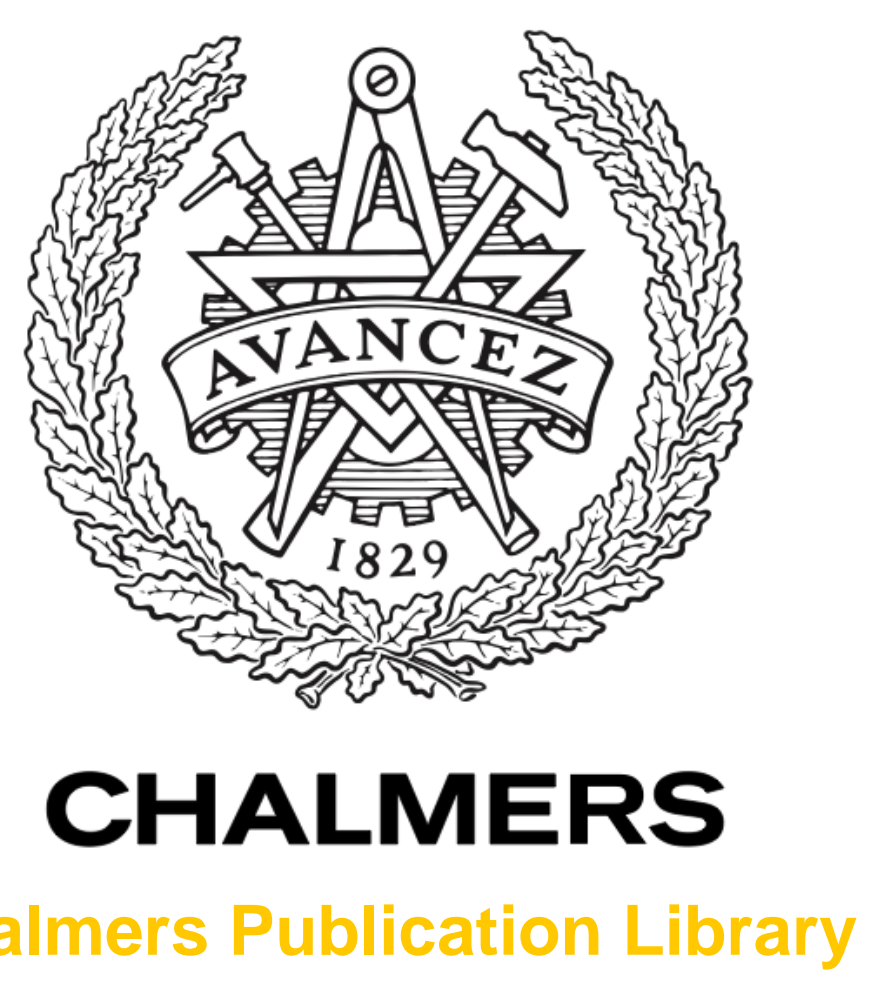

Chalmers Publication Library

\author{
Microwave imaging for breast cancer detection: Comparison of tomographic \\ imaging algorithms using single-frequency and time-domain data
}

This document has been downloaded from Chalmers Publication Library $(\mathrm{CPL})$. It is the author's version of a work that was accepted for publication in:

30th URSI General Assembly and Scientific Symposium, URSIGASS 2011, Istanbul, 13-20 August 2011

Citation for the published paper:

Rubaek, T. ; Fhager, A. ; Jensen, P. (2011) "Microwave imaging for breast cancer detection: Comparison of tomographic imaging algorithms using single-frequency and time-domain data". 30th URSI General Assembly and Scientific Symposium, URSIGASS 2011, Istanbul, 13-20 August 2011

http://dx.doi.org/10.1109/URSIGASS.2011.605137

0

Down「oaded from: http://publications.lib.chalmers.se/publication/151131

Notice: Changes introduced as a result of publishing processes such as copy-editing and formatting may not be reflected in this document. For a definitive version of this work, please refer to the published source. Please note that access to the published version might require a subscription.

Chalmers Publication Library (CPL) offers the possibility of retrieving research publications produced at Chalmers University of Technology. It covers all types of publications: articles, dissertations, licentiate theses, masters theses, conference papers, reports etc. Since 2006 it is the official tool for Chalmers official publication statistics. To ensure that Chalmers research results are disseminated as widely as possible, an Open Access Policy has been adopted.

The CPL service is administrated and maintained by Chalmers Library. 


\title{
Microwave Imaging for Breast Cancer Detection: Comparison of Tomographic Imaging Algorithms using Single-Frequency and Time-Domain Data
}

\author{
Tonny Rubak ${ }^{1}$, Andreas Fhager ${ }^{1}$, Peter Damsgaard Jensen ${ }^{2}$, Johan Jacob Mohr ${ }^{2}$, and \\ Mikael Persson ${ }^{1}$ \\ ${ }^{1}$ Division of Signal Processing and Biomedical Engineering, Department of Signals and Systems \\ Chalmers University of Technology \\ SE-41296 Gothenburg, Sweden \\ rubaek@chalmers.se, andreas.fhager@chalmers.se, mikael.persson@chalmers.se \\ 2 Department of Electrical Engineering \\ Technical University of Denmark \\ DK-2800 Kgs. Lyngby, Denmark \\ pdj@elektro.dtu.dk,jm@elektro.dtu.dk
}

\begin{abstract}
Still more research groups are promoting microwave imaging as a viable supplement or substitution to more conventional imaging modalities. A widespread approach for microwave imaging of the breast is tomographic imaging in which one seeks to reconstruct the distributions of permittivity and conductivity in the breast. In this paper two nonlinear tomographic algorithms are compared - one is a single-frequency algorithm and the other is a time-domain algorithm.
\end{abstract}

\section{Introduction}

An increasing number of research groups have turned their attention toward developing microwave-based imaging systems for breast-cancer diagnostics. The use of microwaves for imaging of the breast is motivated by the contrast in constitutive electromagnetic parameters, i.e., permittivity and conductivity, between the healthy breast tissue and cancerous tumors which as been reported by several authors, e.g. [1]. This contrast implies that the presence of a tumor in the breast will cause an incident electromagnetic field to scatter and by observing the resulting field, the location of the tumor can be determined.

Different approaches have been used for microwave imaging of which the two most widespread are the radar-based approach [2] and the tomographic approach [3-5]. In the radar-based algorithms, the imaging problem is treated as a linear inverse problem and the resulting images indicates the points of origin for the reflected signals of the incident ultra-wideband pulse used to illuminate the breast.

The tomography-based approaches differ from the radar-based approaches in that they seek to reconstruct the distribution of the constitutive parameters of the breast. This is usually done iteratively and thus requires significantly more computational power than the radar-based approaches. However, these methods also seem to better at handling large contrasts between the parameters in the breast, thus leading to algorithms which can be used for a wider range of breast types.

Different tomographic techniques have been suggested for imaging of the breast, including single-frequency, multi-frequency, and time-domain tomography. These algorithms have different requirements to the hardware of the imaging system as well as to the computational power needed to create images. In this paper a single-frequency (SF) tomographic algorithm is compared with a time-domain (TD) tomographic algorithm. While the requirements to the imaging hardware and the computational power is less for the SF algorithm, the TD algorithm has the advantage of collecting more information about the object since the signals used in this algorithm cover a large frequency band. 


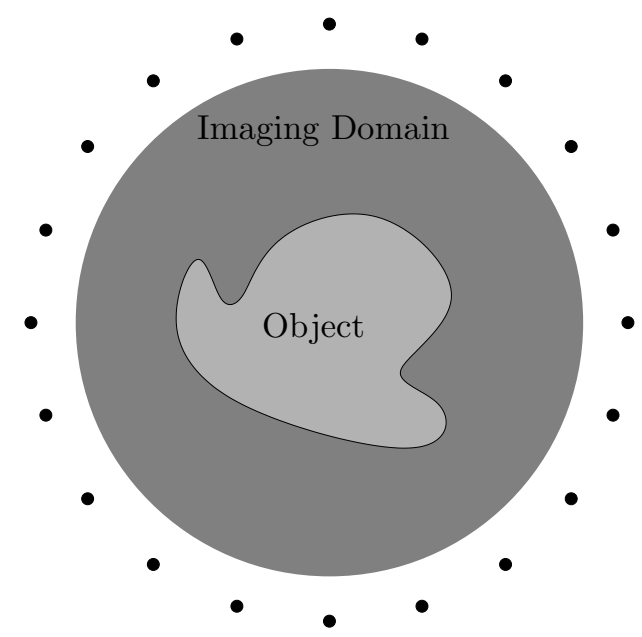

Figure 1: Schematic of imaging system. The solid black dots indicate the positions of the antennas while the dark-gray area indicates the position of the imaging domain.

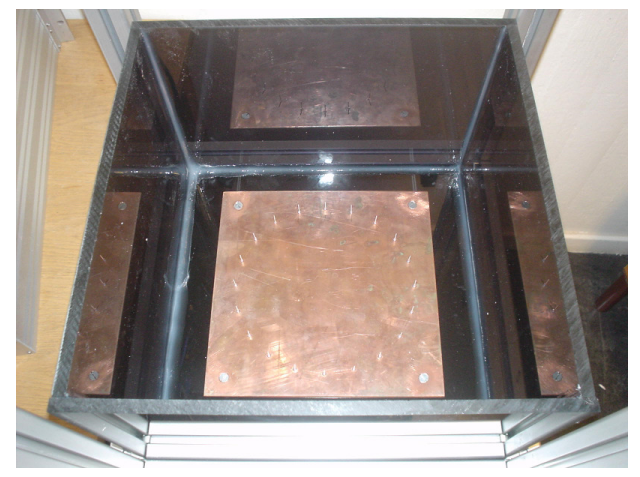

Figure 2: Photo of the imaging system. The 20 monopole antennas are mounted on a copper ground plane.

\section{Imaging Algorithms}

In this paper, the two imaging algorithms to be compared are both used on a simulated two-dimensional imaging system similar to the imaging system which has been tested at Chalmers University of Technology. A schematic of the system is shown in Figure 1 and a photo of the system is shown in Figure 2. The system consists of 20 antennas in a circular setup with a radius of $10 \mathrm{~cm}$ and the imaging domain, in which the object to be imaged is positioned, has a radius of $8 \mathrm{~cm}$.

When performing measurements with the imaging system, each of the 20 antennas are in turn used as a transmitter while the remaining 19 antennas are used as receivers. This leads to a total of 380 measurements of either complex S-parameters (for the single-frequency algorithm) or real-valued time signals (for the TD algorithm).

\subsection{Single-Frequency Imaging Algorithm}

The single-frequency imaging algorithm used in this paper is described in details in [5] and is based on solving the minimization problem

$$
\left[\underline{k}^{2}\right]=\operatorname{argmin}\left\{\left\|\underline{E}^{\text {meas }}-\underline{E}^{\text {calc }}\left(\underline{k}^{2}\right)\right\|_{2}^{2}\right\}=\operatorname{argmin}\left\{\left\|\underline{E}^{\mathrm{res}}\left(\underline{k}^{2}\right)\right\|_{2}^{2}\right\}
$$

using an iterative Newton-type algorithm.

In (1), the vector $\underline{k}^{2}$ holds the squared complex wave numbers

$$
k^{2}(\boldsymbol{r})=\mu_{0} \omega^{2} \epsilon(\boldsymbol{r})+i \mu_{0} \omega \sigma(\boldsymbol{r})
$$

of the individual cells of the discretized imaging domain. In this paper, the imaging domain will be divided into square cells with a side length of $2 \mathrm{~mm}$, yielding a total of 4849 cells.

The vectors $\underline{S}^{\text {meas }}$ and $\underline{S}^{\text {calc }}$ holds the measured and calculated S-parameters for the system in the log-phase formulation [3] while $\underline{E}^{\text {res }}(\underline{\epsilon})$ is the residual vector. 


\subsection{Time-Domain Imaging Algorithm}

The time-domain imaging algorithm used in this algorithm is described in details in [4] and will only be briefly described here. The algorithm is based on finding the solution $\underline{k}^{2}$ to the minimization problem

$$
\underline{k}^{2}=\operatorname{argmin}\left\{\int_{0}^{T} \sum_{T x=1}^{20} \sum_{R x=1}^{20}\left|S_{R x, T x}^{\mathrm{calc}}\left(t^{\prime}, \underline{k}^{2}\right)-S_{R x, T x}^{\text {meas }}\left(t^{\prime}\right)\right|^{2} \mathrm{~d} t^{\prime}\right\} .
$$

As is the case in (1), the vector $\underline{k}^{2}$ holds the constitutive parameters of the individual cells in the imaging domain in form of the squared complex wave numbers of the domain. The imaging domain is again divided into 4849 square cells with a side length of $2 \mathrm{~mm}$.

Although, in theory, any pulse can be used in the time-domain algorithm, it has been found that a Gaussian pulse is often the best choice. Such a pulse is characterized by a certain center frequency $f_{\mathrm{c}}$ and a certain full-width half-maximum bandwidth $f_{\mathrm{FWHM}}$. In this case it has been found that the total span of frequencies needed to adequately represent the pulse is from approximately $f_{\mathrm{c}}-f_{\mathrm{FWHM}}$ to approximately $f_{\mathrm{c}}+f_{\mathrm{FWHM}}$. Hence, for the TD algorithm to perform optimally, the hardware (data acquisition system and antennas) should be capable to function in this frequency span. This is a much more stringent requirement to the hardware than the requirements of the SF algorithm in which the hardware only needs to perform well at a single frequency.

\section{Results}

To illustrate the difference in performance between the two algorithms, a simple breast model has been simulated. The breast model, which is shown in Figures 3(a) and 3(d), consists of a circular region with a radius of $4.5 \mathrm{~cm}$ in which two smaller circular regions with radius $1.0 \mathrm{~cm}$ and $0.8 \mathrm{~cm}$ have been inserted. The large circular region has a relative permittivity of 10 and a conductivity of $0.4 \mathrm{~S} / \mathrm{m}$ while the two insertions both have a relative permittivity of 60 and a conductivity of $1.2 \mathrm{~S} / \mathrm{m}$. The background is assumed to be uniform and has a relative permittivity of 20 and a conductivity of $0.8 \mathrm{~S} / \mathrm{m}$. All of the parameters are assumed constant over frequency.

The actual distributions of the permittivity and conductivity as well as the distributions reconstructed with the SF and TD algorithms are shown in Figure 3. In these reconstructions a Gaussian pulse with $f_{\mathrm{c}}=1.2 \mathrm{GHz}$ and $f_{\mathrm{FWHM}}=1.0 \mathrm{GHz}$ is used in the TD algorithm while the SF algorithm uses the frequency 1.7 GHz (corresponding to the center frequency plus half the FWHM bandwidth).

Both algorithms are seen to reconstruct the large circular region and the two small insertions quite well in the permittivity image while the conductivity images are somewhat worse.

\section{Conclusion}

Two different algorithms for microwave imaging have been compared. It is seen that the images are similar, with the TD algorithm providing smoother images with less artifacts than the SF algorithm.

At the conference, more results will be presented and discussed in details, including images reconstructed from data acquired from measurements with both 2D and 3D imaging systems. 


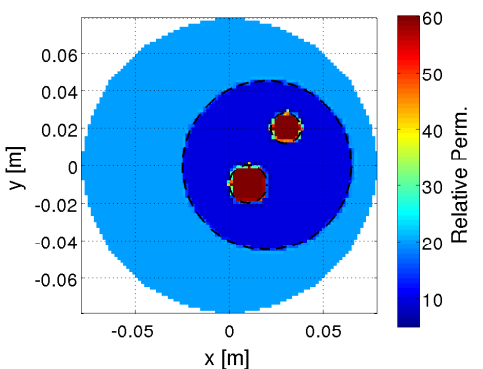

(a) True permittivity

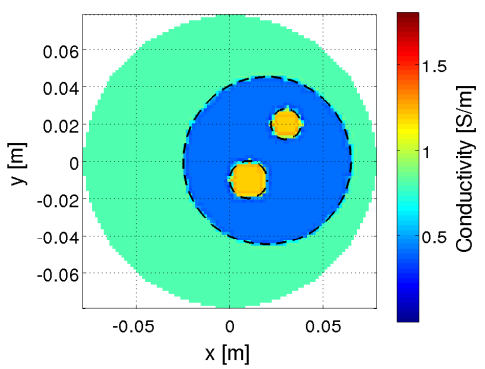

(d) True conductivity

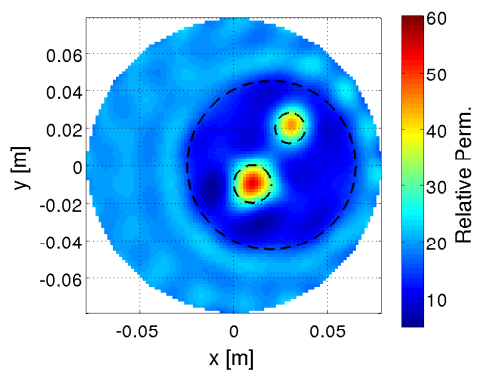

(b) Single frequency

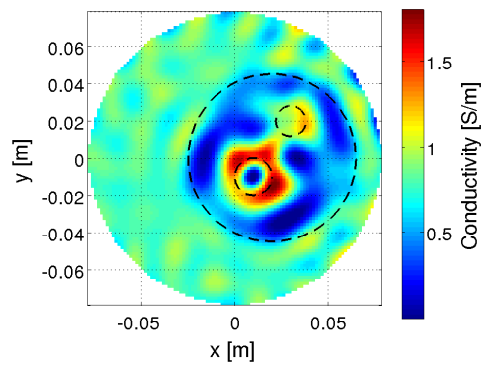

(e) Single frequency

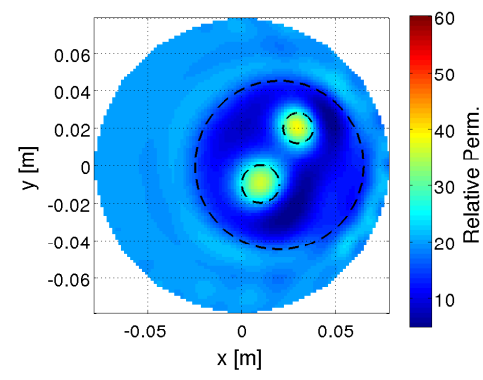

(c) Time domain

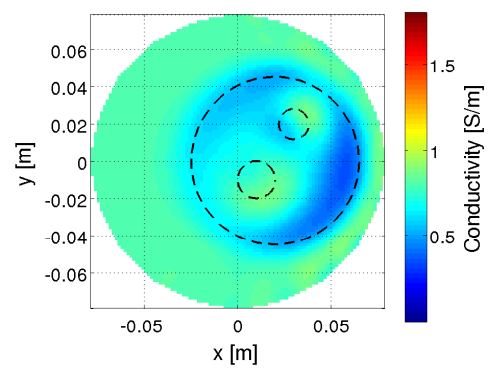

(f) Time domain

Figure 3: Reconstructed images. The actual distribution of the permittivity and conductivity is shown in (a) and (d). In (b) and (c), the permittivity reconstructed with the SF and TD algorithms, respectively, are shown. The corresponding conductivity images are shown in (e) and (f). The dashed lines indicate the position of the three circular regions.

\section{References}

[1] M. Lazebnik, D. Popovic, L. McCartney, C. B. Watkins, M. J. Lindstrom, J. Harter, S. Sewall, T. Ogilvie, A. Magliocco, T. M. Breslin, W. Temple, D. Mew, J. H. Booske, M. Okoniewski, and S. C. Hagness, "A large-scale study of the ultrawideband microwave dielectric properties of normal, benign and malignant breast tissues obtained from cancer surgeries," Physics in Medicine and Biology, vol. 52, no. 20, pp. 6093-6115, 2007.

[2] T. C. Williams, J. M. Sill, and E. C. Fear, "Breast surface estimation for Radar-Based breast imaging systems," IEEE Transactions on Biomedical Engineering, vol. 55, no. 6, pp. 1678-1686, 2008. [Online]. Available: http://ieeexplore.iee.org/lpdocs/epic03/wrapper.htm?arnumber $=4524032$

[3] P. M. Meaney, K. D. Paulsen, B. W. Pogue, and M. I. Miga, "Microwave image reconstruction utilizing log-magnitude and unwrapped phase to improve high-contrast object recovery," Medical Imaging, IEEE Transactions on, vol. 20, no. 2, pp. 104-116, 2001.

[4] A. Fhager, S. Padhi, and J. Howard, "3D image reconstruction in microwave tomography using an efficient FDTD model," Antennas and Wireless Propagation Letters, IEEE, vol. 8, pp. 1353-1356, 2009.

[5] T. Rubaek, O. S. Kim, and P. Meincke, "Computational validation of a 3-D microwave imaging system for Breast-Cancer screening," IEEE Transactions on Antennas and Propagation, vol. 57, no. 7, pp. 2105$2115,2009$. 